\title{
Analysis of Some Finite Elements for the Stokes Problem
}

\section{By Christine Bernardi and Geneviève Raugel}

\begin{abstract}
We study some finite elements which are used in the approximation of the Stokes problem, so as to obtain error estimates of optimal order.

Résumé. Nous étudions deux éléments finis utilisés pour l'approximation du problème de Stokes et obtenons des estimations d'erreur d'ordre optimal.
\end{abstract}

I. Introduction. Let $\Omega$ be a bounded polyhedral domain in $\mathbf{R}^{d}, d=2$ or 3 . We consider the standard variational formulation of the stationary Stokes equations: for f given in $H^{-1}(\Omega)^{d}$, find $(\mathbf{u}, p)$ in $H_{0}^{1}(\Omega)^{d} \times L_{0}^{2}(\Omega)$ such that

$$
\left\{\begin{array}{l}
\forall \mathbf{v} \in H_{0}^{1}(\Omega)^{d}, \quad \nu(\operatorname{grad} \mathbf{u}, \operatorname{grad} \mathbf{v})-(p, \operatorname{div} \mathbf{v})=(\mathbf{f}, \mathbf{v}), \\
\forall q \in L_{0}^{2}(\Omega), \quad(q, \operatorname{div} \mathbf{u})=0,
\end{array}\right.
$$

where we denote by $(\cdot, \cdot)$ the inner product of $L^{2}(\Omega)$ (or $L^{2}(\Omega)^{d}$ or $L^{2}(\Omega)^{d^{2}}$ ). Hereafter $L_{0}^{2}(\Omega)$ is the space $\left\{q \in L^{2}(\Omega) ; \int_{\Omega} q d x=0\right\}$. Now let $h$ be a real positive parameter tending to zero. We introduce two finite-dimensional subspaces $X_{h}$ and $M_{h}$ of $H_{0}^{1}(\Omega)^{d}$ and $L_{0}^{2}(\Omega)$ respectively, satisfying the usual condition: for any $q_{h}$ in $M_{h}, q_{h} \neq 0$, there exists $\mathbf{v}_{h}$ in $X_{h}$ such that $\left(q_{h}, \operatorname{div} \mathbf{v}_{h}\right) \neq 0$. We consider the discretized problem: find $\left(\mathbf{u}_{h}, p_{h}\right)$ in $X_{h} \times M_{h}$ such that

$$
\left\{\begin{array}{l}
\forall \mathbf{v}_{h} \in X_{h}, \quad \nu\left(\operatorname{grad} \mathbf{u}_{h}, \operatorname{grad} \mathbf{v}_{h}\right)-\left(p_{h}, \operatorname{div} \mathbf{v}_{h}\right)=\left(\mathbf{f}, \mathbf{v}_{h}\right), \\
\forall q_{h} \in M_{h}, \quad\left(q_{h}, \operatorname{div} \mathbf{u}_{h}\right)=0 .
\end{array}\right.
$$

We recall that problem (I.1) (respectively problem (I.2)) has a unique solution (u, $p$ ) in $H_{0}^{1}(\Omega)^{d} \times L_{0}^{2}(\Omega)$ (respectively $\left(\mathbf{u}_{h}, p_{h}\right)$ in $\left.X_{h} \times M_{h}\right)$. Moreover, when $(\mathbf{u}, p)$ belongs to the space $H^{m+1}(\Omega)^{d} \times H^{m}(\Omega)$, it is well-known (see [7]) that the error estimate

$$
\left\|\mathbf{u}-\mathbf{u}_{h}\right\|_{1, \Omega}+\left\|p-p_{h}\right\|_{0, \Omega} \leqslant C h^{m}\left(\|\mathbf{u}\|_{m+1, \Omega}+\|p\|_{m, \Omega}\right)
$$

holds whenever the following additional hypotheses are satisfied:

(H1) for any $q$ in $H^{m}(\Omega) \cap L_{0}^{2}(\Omega)$, one has

$$
\operatorname{Inf}_{q_{h} \in M_{h}}\left\|q-q_{h}\right\|_{0, \Omega} \leqslant C h^{m}\|q\|_{m, \Omega}
$$

(H2) there exists a linear operator $\Pi_{h}$ from $H^{m+1}(\Omega)^{d} \cap H_{0}^{1}(\Omega)^{d}$ into $X_{h}$ such that

$$
\forall \mathbf{v} \in H^{m+1}(\Omega)^{d} \cap H_{0}^{1}(\Omega)^{d}, \quad\left\{\begin{array}{l}
\forall q_{h} \in M_{h}, \quad\left(q_{h}, \operatorname{div}\left(\mathbf{v}-\Pi_{h} \mathbf{v}\right)\right)=0, \\
\left\|\mathbf{v}-\Pi_{h} \mathbf{v}\right\|_{1, \Omega} \leqslant C h^{m}\|\mathbf{v}\|_{m+1, \Omega} ;
\end{array}\right.
$$

Received May 24, 1983.

1980 Mathematics Subject Classification. Primary 65N30. 
(H3) for each $q_{h}$ in $M_{h}$, there exists a function $\mathbf{v}_{h}$ in $X_{h}$ such that

$$
\left(\operatorname{div} \mathbf{v}_{h}, q_{h}\right) \geqslant \beta\left\|q_{h}\right\|_{0, \Omega}\left\|\mathbf{v}_{h}\right\|_{1, \Omega},
$$

where $\beta>0$ is a constant independent of $h$.

Our aim is to give some examples of finite-element spaces such that hypotheses (H1), (H2) and (H3) are satisfied. To this end, we introduce a family $\left(\mathscr{T}_{h}\right)_{h}$ of triangulations of $\bar{\Omega}$, where $\mathscr{T}_{h}$ is made of $d$-simplices with diameters bounded by $h$.

For any integer $k, P_{k}(K)$ denotes the space of polynomials of degree $\leqslant k$ on $K$. We set

$$
M_{h}^{(m)}=\left\{q_{h} \in L_{0}^{2}(\Omega) ; \forall K \in \mathscr{T}_{h}, q_{h / K} \in P_{m-1}(K)\right\} .
$$

Then hypothesis (H1) is satisfied (see [2] for instance). Finally, we set

$$
X_{h}=\left\{\mathbf{v}_{h} \in \mathscr{C}^{0}(\bar{\Omega})^{d} \cap H_{0}^{1}(\Omega)^{d} ; \forall K \in \mathscr{T}_{h}, \mathbf{v}_{h / K} \in P_{K}\right\} ;
$$

hereafter we study some examples of spaces $P_{K}$ introduced by Fortin [6] such that hypotheses (H2) and (H3) are satisfied.

More precisely, we give in Section II an example of a simplicial element of order $m=1$ and, in Section III, an example of a three-dimensional tetrahedral element of order $m=2$.

From now on we denote by $\|\cdot\|_{m, \Omega}$ and $|\cdot|_{m, \Omega}$ the usual norm and seminorm on the Sobolev space $H^{m}(\Omega)$.

II. A Simplicial Element of Order $1(d=2$ or 3$)$. Let us consider a $d$-simplex $K$ with vertices $a_{1}, \ldots$ and $a_{d+1}$. For $1 \leqslant i \leqslant d+1$, we denote by $\lambda_{i}$ the barycentric coordinate associated with $a_{i}$, by $F_{i}$ the face which does not contain $a_{i}$, and by $\mathbf{n}_{i}$ the unit outward normal to $F_{i}$, and we set

$$
\mathbf{p}_{i}=\mathbf{n}_{i} \prod_{j=1, j \neq i}^{d+1} \lambda_{j} .
$$

Then, we consider

$$
P_{K}=P_{1}(K)^{d} \oplus \operatorname{Span}\left\{\mathbf{p}_{i}, 1 \leqslant i \leqslant d+1\right\} .
$$

(Note that $\operatorname{dim} P_{K}=(d+1)^{2}$.) As far as the degrees of freedom are concerned, we can choose the values at the vertices $a_{i}, 1 \leqslant i \leqslant d+1$, and the flux through the faces $F_{i}, 1 \leqslant i \leqslant d+1$.

LEMMA II.1. For any $\mathbf{v}$ in $\mathscr{C}^{0}(K)^{d}$, there exists a unique $\Pi_{K} \mathbf{v}$ in $P_{K}$ such that

$$
\left\{\begin{array}{l}
\Pi_{K} \mathbf{v}\left(a_{i}\right)=\mathbf{v}\left(a_{i}\right), \\
\int_{F_{i}}\left(\mathbf{v}-\Pi_{K} \mathbf{v}\right) \cdot \mathbf{n}_{i} d \boldsymbol{\sigma}=0, \quad 1 \leqslant i \leqslant d+1 .
\end{array}\right.
$$

Moreover, $\Pi_{K} \mathbf{v}_{/ F_{i}}$ depends only on $\mathbf{v}_{/ F_{i}}, 1 \leqslant i \leqslant d+1$.

Proof. Let us denote by $\tilde{\Pi}_{K} \mathbf{v}$ the classical Lagrange interpolate of $\mathbf{v}$ in $P_{1}(K)^{d}$, i.e.,

$$
\tilde{\Pi}_{K} \mathbf{v}=\sum_{i=1}^{d+1} \mathbf{v}\left(a_{i}\right) \lambda_{i}
$$


Then, as the $\mathbf{p}_{i}$ 's are equal to 0 at any vertex, one has

$$
\left\{\begin{aligned}
\Pi_{K} \mathbf{v}=\tilde{\Pi}_{K} \mathbf{v} & +\sum_{i=1}^{d+1} \alpha_{i} \mathbf{p}_{i} \\
& \text { with } \alpha_{i}=\left(\int_{F_{i}}\left(\mathbf{v}-\tilde{\Pi}_{K} \mathbf{v}\right) \cdot \mathbf{n}_{i} d \boldsymbol{\sigma}\right) / \int_{F_{i}} \prod_{j=1, j \neq i}^{d+1} \lambda_{j} d \boldsymbol{\sigma} .
\end{aligned}\right.
$$

Moreover, on $F_{i}$,

$$
\Pi_{K} \mathbf{v}_{/ F_{i}}=\sum_{j=1, j \neq i}^{d+1} \mathbf{v}\left(a_{j}\right) \lambda_{j}+\alpha_{i} \mathbf{p}_{i}
$$

so that $\Pi_{K} \mathbf{v}_{/ F_{i}}$ depends only on $\mathbf{v}\left(a_{j}\right), j \neq i$, and on $\int_{F_{i}} \mathbf{v} \cdot \mathbf{n}_{i} d \boldsymbol{\sigma}$.

Now, for each $h$, we consider a triangulation $\mathscr{T}_{h}$ of $\bar{\Omega}$ made of $d$-simplices with diameters bounded by $h$ and we assume that the family $\left(\mathscr{T}_{h}\right)_{h}$ is regular, i.e., (see [2]) there exists a constant $\sigma$ such that

$$
\forall h, \forall K \in \mathscr{T}_{h}, \quad h_{K} \leqslant \sigma \rho_{K},
$$

where $h_{K}$ is the diameter of $K$, and $\rho_{K}$ the diameter of the sphere inscribed in $K$.

With each $K$ in $\mathscr{T}_{h}$, we associate the space $P_{K}$ defined by (II.1); then Lemma II.1 allows us to define an operator $\Pi_{h}$ from $\mathscr{C}^{0}(\bar{\Omega})^{d} \cap H_{0}^{1}(\Omega)^{d}$ into $X_{h}$ by

$$
\forall K \in \mathscr{T}_{h}, \quad \Pi_{h} \mathbf{v}_{/ K}=\Pi_{K} \mathbf{v} .
$$

LEMMA II.2. The operator $\Pi_{h}$ satisfies $(\mathrm{H} 2)$ for $m=1$.

Proof. Clearly, one has

$$
\int_{K} \operatorname{div}\left(\mathbf{v}-\Pi_{K} \mathbf{v}\right) d x=\sum_{i=1}^{d+1} \int_{F_{i}}\left(\mathbf{v}-\Pi_{K} \mathbf{v}\right) \cdot \mathbf{n}_{i} d \boldsymbol{\sigma}=0,
$$

so that $\forall q_{h} \in M_{h}^{(1)},\left(q_{h}, \operatorname{div}\left(\mathbf{v}-\Pi_{h} \mathbf{v}\right)\right)=0$.

Moreover, we know that (see [2], for instance), for $k=0$ and 1,

$$
\left|\mathbf{v}-\tilde{\Pi}_{K} \mathbf{v}\right|_{k, K} \leqslant C h^{2-k}|\mathbf{v}|_{2, K} .
$$

Let us compute $\Pi_{K} \mathbf{v}-\tilde{\Pi}_{K} \mathbf{v}=\sum_{i=1}^{d+1} \alpha_{i} \mathbf{p}_{i}$. We consider an affine invertible mapping $F_{K}: \hat{x} \mapsto x=B_{K} \hat{x}+b_{K}$ which maps the $d$-simplex $\hat{K}=\left\{\hat{x} \in \mathbf{R}^{d} ; \forall i, 1 \leqslant i \leqslant d\right.$, $\hat{x}_{i} \geqslant 0$ and $\left.\sum_{i=1}^{d} \hat{x}_{i} \leqslant 1\right\}$ onto $K$, and use the notations $x=F_{K}(\hat{x}), v=\hat{v} \circ F_{K}^{-1}$. Clearly, one has

$$
\begin{aligned}
\left|\mathbf{p}_{i}\right|_{k, K}^{2} & =\int_{K}\left\|D^{k}\left(\prod_{j=1, j \neq i}^{d+1} \lambda_{j}\right)\right\|^{2} d x \\
& \leqslant C \int_{\hat{K}}\left\|D^{k}\left(\prod_{j=1, j \neq i}^{d+1} \hat{\lambda}_{j}\right)\right\|^{2}\left\|B_{K}^{-1}\right\|^{2 k}\left|\operatorname{det} B_{K}\right| d \hat{x} \leqslant C\left|\operatorname{det} B_{K}\right|\left\|B_{K}\right\|^{-2 k}
\end{aligned}
$$

so that, by the regularity of the family $\left(\mathscr{T}_{h}\right)_{h}$,

$$
\left|\mathbf{p}_{i}\right|_{k, K} \leqslant C h_{K}^{d / 2-k} \text {. }
$$

But, since

$$
\int_{F_{i}} \prod_{j=1, j \neq i}^{d+1} \lambda_{j} d \sigma=\left|\operatorname{det} B_{K / \hat{F}_{i}}\right| \int_{\hat{F}_{i}} \prod_{j=1, j \neq i}^{d+1} \hat{\lambda}_{j} d \hat{\sigma}
$$


we obtain by (II.3)

$$
\left|\boldsymbol{\alpha}_{i}\right| \leqslant C\left|\operatorname{det} B_{K / \hat{F}_{i}}\right|^{-1} \int_{F_{i}}\left|\mathbf{v}-\tilde{\Pi}_{K} \mathbf{v}\right| d \sigma \leqslant C \int_{\hat{F}_{i}}\left|\hat{\mathbf{v}}-\hat{\tilde{\Pi}}_{\hat{K}} \hat{\mathbf{v}}\right| d \hat{\sigma} ;
$$

therefore, as $P_{1}(\hat{K})^{d}$ is invariant under $\hat{\tilde{\Pi}}_{\hat{K}}$,

$$
\left|\boldsymbol{\alpha}_{i}\right| \leqslant C|\hat{\mathbf{v}}|_{2, \hat{K}}^{-} \leqslant C\left|\operatorname{det} B_{K}\right|^{-1 / 2}\left\|B_{K}\right\|^{2}|\mathbf{v}|_{2, K} \leqslant C h_{K}^{2-d / 2}|\mathbf{v}|_{2, K}
$$

The previous inequalities yield, for $k=0$ and 1 ,

$$
\left|\mathbf{v}-\Pi_{K} \mathbf{v}\right|_{k, K} \leqslant C h_{K}^{2-k}|\mathbf{v}|_{2, K},
$$

so that

$$
\left\|\mathbf{v}-\Pi_{K} \mathbf{v}\right\|_{1, \Omega} \leqslant C h|\mathbf{v}|_{2, \Omega} .
$$

We recall the proof of the following inequality only for the reader's convenience.

LEMMA II.3. For any $v$ in $H^{1}(K)$, we have

$$
\|v\|_{0, F_{i}} \leqslant C\left|\operatorname{mes} F_{i}\right|^{1 / 2} h_{K}^{-d / 2}\left\{\|v\|_{0, K}+h_{K}|v|_{1, K}\right\} \text {. }
$$

Proof. As the trace mapping is continuous from $H^{1}(\hat{K})$ into $L^{2}\left(\hat{F}_{i}\right)$,

$$
\begin{aligned}
\|v\|_{0, F_{i}}^{2} & =\left|\operatorname{det} B_{K / \hat{F}_{i}}\right| \int_{\hat{F}_{i}} \hat{v}^{2} d \hat{\sigma} \leqslant C\left|\operatorname{det} B_{K / \hat{F}_{i}}\right|\left\{\|\hat{v}\|_{0, \hat{K}}^{2}+|\hat{v}|_{1, \hat{K}}^{2}\right\} \\
& \leqslant C\left|\operatorname{mes} F_{i}\right| h_{K}^{-d}\left\{\|v\|_{0, K}^{2}+h_{K}^{2}|v|_{1, K}^{2}\right\} .
\end{aligned}
$$

Let us now study the hypothesis (H3). We know (see [7, Chapter I, Lemma 3.2]) that, for each $q_{h}$ in $M_{h}^{(1)}$, there exists $v$ in $H_{0}^{1}(\Omega)^{d}$ such that

$$
\operatorname{div} \mathbf{v}=q_{h} \text { and }\|\mathbf{v}\|_{1, \Omega} \leqslant C\left\|q_{h}\right\|_{0, \Omega} .
$$

Hence, the hypothesis $(\mathrm{H} 3)$ is an immediate consequence of the following

LEMMA II.4. For any $\mathrm{v}$ in $H_{0}^{1}(\Omega)^{d}$, there exists $\mathrm{v}_{h}$ in $X_{h}$ such that

$$
\forall q_{h} \in M_{h}^{(1)}, \quad\left\{\begin{array}{l}
\left(q_{h}, \operatorname{div}\left(\mathbf{v}-\mathbf{v}_{h}\right)\right)=0 \\
\text { and }\left\|\mathbf{v}_{h}\right\|_{1, \Omega} \leqslant C\|\mathbf{v}\|_{1, \Omega} .
\end{array}\right.
$$

Proof. Let us denote by $\mathbf{w}_{h}$ the interpolate of $\mathbf{v}$ in the space

$$
\left\{u_{h} \in \mathscr{C}^{0}(\bar{\Omega}) \cap H_{0}^{1}(\Omega) ; \forall K \in \mathscr{T}_{h}, u_{h / K} \in P_{1}(K)\right\}^{d} \text {, }
$$

defined by local regularization as in [4] (see [1] for an explicit generalization to the case $d=3$ ). By the regularity of the family $\left(\mathscr{T}_{h}\right)_{h}$, we know that the following local interpolation error holds

$$
\left\|\mathbf{v}-\mathbf{w}_{h}\right\|_{0, K}+h_{K}\left|\mathbf{w}_{h}\right|_{1, K} \leqslant C h_{K}\|\mathbf{v}\|_{1, \Delta_{K}},
$$

where $\Delta_{K}$ is the union of all $K^{\prime}$ in $\mathscr{T}_{h}$ such that $K \cap K^{\prime} \neq \varnothing$; moreover, each element of $\mathscr{T}_{h}$ is contained in at most $M$ subsets $\Delta_{K}$, where $M$ is an integer independent of $h$.

Then, we consider the element $\mathbf{v}_{h}$ in $V_{h}$ defined by

$$
\left\{\begin{array}{l}
\mathbf{v}_{h}\left(a_{i}\right)=\mathbf{w}_{h}\left(a_{i}\right), \\
\int_{F_{i}}\left(\mathbf{v}-\mathbf{v}_{h}\right) \cdot \mathbf{n}_{i} d \boldsymbol{\sigma}=0,
\end{array}\right.
$$


or, in other words, equal on $K$ to

$$
\left\{\begin{aligned}
\mathbf{v}_{h / K}=\mathbf{w}_{h}+ & \sum_{i=1}^{d+1} \alpha_{i} \mathbf{p}_{i} \\
& \text { with } \alpha_{i}=\left(\int_{F_{i}}\left(\mathbf{v}-\mathbf{w}_{h}\right) \cdot \mathbf{n}_{i} d \sigma\right) / \int_{F_{i}} \prod_{j=1, j \neq i}^{d+1} \lambda_{j} d \sigma .
\end{aligned}\right.
$$

Clearly, one has $\forall q_{h} \in M_{h}^{(1)},\left(q_{h}, \operatorname{div}\left(\mathbf{v}-\mathbf{v}_{h}\right)\right)=0$. Moreover, by (II.6),

$$
\left\|\mathbf{v}_{h}\right\|_{1, K} \leqslant\left\|\mathbf{w}_{h}\right\|_{1, K}+\sum_{i=1}^{d+1}\left|\alpha_{i}\right|\left\|\mathbf{p}_{i}\right\|_{1, K} \leqslant\left\|\mathbf{w}_{h}\right\|_{1, K}+C h_{K}^{d / 2-1} \sum_{i=1}^{d+1}\left|\alpha_{i}\right| .
$$

But, we also have

$$
\left|\alpha_{i}\right| \leqslant C\left|\operatorname{det} B_{K / \hat{F}_{i}}\right|^{-1} \int_{F_{i}}\left(\mathbf{v}-\mathbf{w}_{h}\right) \cdot \mathbf{n}_{i} d \sigma \leqslant C\left|\operatorname{mes} F_{i}\right|^{-1 / 2}\left\|\mathbf{v}-\mathbf{w}_{h}\right\|_{0, F_{i}} \cdot
$$

Lemma II.3 implies

$$
\left|\alpha_{i}\right| \leqslant C h_{K}^{-d / 2}\left\{\left\|\mathbf{v}-\mathbf{w}_{h}\right\|_{0, K}+h_{K}\left|\mathbf{v}-\mathbf{w}_{h}\right|_{1, K}\right\} .
$$

Finally, we obtain

$$
\left\|\mathbf{v}_{h}\right\|_{1, K} \leqslant\left\|\mathbf{w}_{h}\right\|_{1, K}+h_{K}^{-1}\left\{\left\|\mathbf{v}-\mathbf{w}_{h}\right\|_{0, K}+h_{K}\left|\mathbf{v}-\mathbf{w}_{h}\right|_{1, K}\right\},
$$

which, together with (II.10), yields $\left\|\mathbf{v}_{h}\right\|_{1, \Omega} \leqslant C\|\mathbf{v}\|_{1, \Omega}$.

As assumptions $(\mathrm{H} 1)$ to $(\mathrm{H} 3)$ are satisfied with $m=1$, this element can be used to solve the Stokes problem with an $O(h)$-error estimate.

Remark II.1. In the two-dimensional case, we can also consider a triangulation $\mathscr{T}_{h}$ of $\bar{\Omega}$ made of triangles and convex quadrilaterals. Then, if $K$ is a triangle, the space $P_{K}$ is defined by (II.1). If $K$ is a convex quadrilateral with vertices $a_{1}, \ldots$ and $a_{4}$, there exists an invertible mapping $F_{K}$ in $\hat{Q}_{1}^{2}$ which maps the unit square $\hat{K}=[0,1]^{2}$ onto $K\left(\hat{Q}_{1}\right.$ is the space of polynomials spanned by $\hat{x}_{1}, \hat{x}_{2}, \hat{x}_{3}=1-\hat{x}_{1}$ and $\hat{x}_{4}=1-\hat{x}_{2}$ ); for $1 \leqslant i \leqslant 4$, we denote by $F_{i}$ the edge with vertices $a_{i-1}$ and $a_{i}$ (of course, $a_{0}=a_{4}$ ) and by $\mathbf{n}_{i}$ the unit outward normal to $F_{i}$, and we set

Then, we consider

$$
\mathbf{p}_{i}=\mathbf{n}_{i}\left(\hat{q}_{i} \circ F_{K}^{-1}\right), \quad \hat{q}_{i}=\prod_{j=1, j \neq i}^{4} \hat{x}_{j}
$$

$$
P_{K}=Q_{1}(K)^{2} \oplus \operatorname{Span}\left\{\mathbf{p}_{i}, 1 \leqslant i \leqslant 4\right\}
$$

where $Q_{1}(K)=\left\{\hat{p} \circ F_{K}^{-1}, \hat{p} \in \hat{Q}_{1}\right\}$. (Note that $\operatorname{dim} P_{K}=12$.) The degrees of freedom can be chosen as previously. If the family $\left(\mathscr{T}_{h}\right)_{h}$ is regular (see [3] for instance), the previous results are still valid.

III. A Tetrahedral Element of Order $2(d=3)$. Let us consider a tetrahedron $K$ with vertices $a_{1}, \ldots$ and $a_{4}$. We use the same notations as in Section II, in particular, we set

$$
\mathbf{p}_{i}=\mathbf{n}_{i} \prod_{j=1, j \neq i}^{4} \lambda_{j}, \quad 1 \leqslant i \leqslant 4 ;
$$

we also introduce the points $a_{i j}=\frac{1}{2}\left(a_{i}+a_{j}\right), 1 \leqslant i<j \leqslant 4$. Then, we consider

$$
P_{K}=P_{2}(K)^{3} \oplus \operatorname{Span}\left\{\mathbf{p}_{i}, 1 \leqslant i \leqslant 4\right\} \oplus\left(\operatorname{Span}\left\{\lambda_{1} \lambda_{2} \lambda_{3} \lambda_{4}\right\}\right)^{3}
$$


(Note that $\operatorname{dim} P_{K}=37$.) Let us remark that this space generalizes in the three-dimensional case the space studied in [5] for $d=2$. As far as the degrees of freedom are concerned, we choose the values at the vertices $a_{i}, 1 \leqslant i \leqslant 4$, and at the midpoints $a_{i j}, 1 \leqslant i<j \leqslant 4$, the flux through the faces $F_{i}, 1 \leqslant i \leqslant 4$, and the moments $\int_{K} x_{l} \operatorname{div}(\cdot) d x, 1 \leqslant l \leqslant 3$.

LEMMA III.1. For any $\mathbf{v}$ in $\mathscr{C}^{0}(K)^{3} \cap H^{1}(K)^{3}$, there exists a unique $\Pi_{K} \mathbf{v}$ in $P_{K}$ such that

$$
\begin{cases}\Pi_{K} \mathbf{v}\left(a_{i}\right)=\mathbf{v}\left(a_{i}\right), & 1 \leqslant i \leqslant 4 \\ \Pi_{K} \mathbf{v}\left(a_{i j}\right)=\mathbf{v}\left(a_{i j}\right), & 1 \leqslant i<j \leqslant 4, \\ \int_{F_{i}}\left(\mathbf{v}-\Pi_{K} \mathbf{v}\right) \cdot \mathbf{n}_{i} d \sigma=0, & 1 \leqslant i \leqslant 4 \\ \int_{K} x_{l} \operatorname{div}\left(\mathbf{v}-\Pi_{K} \mathbf{v}\right) d x=0, & 1 \leqslant l \leqslant 3 .\end{cases}
$$

Moreover, $\Pi_{K} \mathbf{v}_{/ F_{i}}$ depends only on $\mathbf{v}_{/ F_{i}}, i \leqslant i \leqslant 4$.

Proof. Let us denote by $\tilde{\Pi}_{K} \mathbf{v}$ the classical Lagrange interpolate of $\mathbf{v}$ in $P_{2}(K)^{3}$, i.e.,

$$
\tilde{\Pi}_{K} \mathbf{v}=\sum_{i=1}^{4} \mathbf{v}\left(a_{i}\right) \lambda_{i}\left(2 \lambda_{i}-1\right)+\sum_{1 \leqslant i<j \leqslant 4} \mathbf{v}\left(a_{i j}\right) 4 \lambda_{i} \lambda_{j} .
$$

Then, as the $\mathbf{p}_{i}$ 's and $\lambda_{1} \lambda_{2} \lambda_{3} \lambda_{4}$ are equal to 0 on any edge, $\Pi_{K} \mathbf{v}$ can be written

$$
\Pi_{K} \mathbf{v}=\tilde{\Pi}_{K} \mathbf{v}+\sum_{i=1}^{4} \alpha_{i} \mathbf{p}_{i}+\beta \lambda_{1} \lambda_{2} \lambda_{3} \lambda_{4} \text {. }
$$

Since $\lambda_{1} \lambda_{2} \lambda_{3} \lambda_{4}$ is equal to 0 on $\partial K$, we have

$$
\alpha_{i}=\left(\int_{F_{i}}\left(\mathbf{v}-\tilde{\Pi}_{K} \mathbf{v}\right) \cdot \mathbf{n}_{i} d \sigma\right) / \int_{F_{i}} \prod_{j=1, j \neq i}^{4} \lambda_{j} d \sigma, \quad 1 \leqslant i \leqslant 4 .
$$

Then, setting

$$
\bar{\Pi}_{K} \mathbf{v}=\tilde{\Pi}_{K} \mathbf{v}+\sum_{i=1}^{4} \alpha_{i} \mathbf{p}_{i}
$$

and using the Green's formula, we obtain

$$
\beta_{l}=-\left(\int_{K} x_{l} \operatorname{div}\left(\mathbf{v}-\bar{\Pi}_{K} \mathbf{v}\right) d x\right) / \int_{K} \lambda_{1} \lambda_{2} \lambda_{3} \lambda_{4} d x, \quad 1 \leqslant l \leqslant 3 .
$$

Moreover, on $F_{i}$, one has

$$
\Pi_{K} \mathbf{v}_{/ F_{i}}=\tilde{\Pi}_{K} \mathbf{v}_{/ F_{i}}+\alpha_{i} \mathbf{p}_{i}
$$

so that $\Pi_{K} \mathbf{v}_{/ F_{i}}$ depends only on $\mathbf{v}_{/ F_{i}}$.

Now, for each $h$, we consider a triangulation $\mathscr{T}_{h}$ of $\bar{\Omega}$ made of tetrahedra with diameters bounded by $h$ and we assume that the family $\left(\mathscr{T}_{h}\right)_{h}$ is regular.

With each $K$ in $\mathscr{T}_{h}$, we associate the space $P_{K}$ defined by (III.1); then Lemma III.1 allows us to define an operator $\Pi_{h}$ from $\mathscr{C}^{0}(\bar{\Omega})^{3} \cap H_{0}^{1}(\Omega)^{3}$ into $X_{h}$ by (II.5).

LEMMA III. 2. The operator $\Pi_{h}$ satisfies $(\mathrm{H} 2)$ for $m=2$. 
Proof. Clearly, one has

$$
\int_{K} \operatorname{div}\left(\mathbf{v}-\Pi_{K} \mathbf{v}\right) d x=\int_{K} x_{l} \operatorname{div}\left(\mathbf{v}-\Pi_{K} \mathbf{v}\right) d x=0, \quad 1 \leqslant l \leqslant 3,
$$

so that $\forall q_{h} \in M_{h}^{(2)},\left(q_{h}, \operatorname{div}\left(\mathbf{v}-\Pi_{h} \mathbf{v}\right)\right)=0$.

Moreover, we know that (see [2]), for $k=0$ and 1,

$$
\left|\mathbf{v}-\tilde{\Pi}_{K} \mathbf{v}\right|_{k, K} \leqslant C h_{K}^{3-k}|\mathbf{v}|_{3, K}
$$

Let us compute $\bar{\Pi}_{K} \mathbf{v}-\tilde{\Pi}_{K} \mathbf{v}=\sum_{i=1}^{4} \alpha_{i} \mathbf{p}_{i}$. As in Section II,

$$
\left|\alpha_{i}\right| \leqslant C \int_{\hat{F}_{i}}\left|\hat{\mathbf{v}}-\hat{\tilde{\Pi}}_{\hat{K}} \hat{\mathbf{v}}\right| d \hat{\sigma}
$$

therefore, as $P_{2}(\hat{K})^{3}$ is invariant under $\hat{\tilde{\Pi}}_{\hat{K}}$,

$$
\left|\alpha_{i}\right| \leqslant C|\hat{\mathbf{v}}|_{3, \hat{K}} \leqslant C h_{K}^{3 / 2}|\mathbf{v}|_{3, K}
$$

The previous inequalities, together with (II.6), yield

$$
\left|\mathbf{v}-\bar{\Pi}_{K} \mathbf{v}\right|_{k, K} \leqslant C h_{K}^{3-k}|\mathbf{v}|_{3, K}
$$

Finally, we compute $\Pi_{K} \mathbf{v}-\bar{\Pi}_{K} \mathbf{v}=\beta \lambda_{1} \lambda_{2} \lambda_{3} \lambda_{4}$. Clearly, one has

$$
\begin{aligned}
\left|\lambda_{1} \lambda_{2} \lambda_{3} \lambda_{4}\right|_{k, K} & \leqslant C\left(\int_{\hat{K}}\left\|D^{k}\left(\hat{\lambda}_{1} \hat{\lambda}_{2} \hat{\lambda}_{3} \hat{\lambda}_{4}\right)\right\|^{2}\left\|B_{K}\right\|^{2 k}\left|\operatorname{det} B_{K}\right| d \hat{x}\right)^{1 / 2} \\
& \leqslant C h_{K}^{3 / 2-k}
\end{aligned}
$$

and, by (III.6),

$$
\left|\beta_{l}\right| \leqslant C\left|\operatorname{det} B_{K}\right|^{-1}\left|\int_{K} x_{l} \operatorname{div}\left(\mathbf{v}-\bar{\Pi}_{K} \mathbf{v}\right) d x\right| .
$$

We use Green's formula

$$
\begin{aligned}
\left|\beta_{l}\right| & \leqslant C\left|\operatorname{det} B_{K}\right|^{-1}\left\{\left|\int_{K}\left(\mathbf{v}-\bar{\Pi}_{K} \mathbf{v}\right)_{l} d x\right|+\left|\int_{\partial K} x_{l}\left(\mathbf{v}-\bar{\Pi}_{K} \mathbf{v}\right) \cdot \mathbf{n} d \sigma\right|\right\} \\
& \leqslant C\left\{\left|\operatorname{det} B_{K}\right|^{-1 / 2}\left\|\mathbf{v}-\bar{\Pi}_{K} \mathbf{v}\right\|_{0, K}+\left|\operatorname{det} B_{K}\right|^{-1}\left|\int_{\partial K} x_{l}\left(\mathbf{v}-\bar{\Pi}_{K} \mathbf{v}\right) \cdot \mathbf{n} d \sigma\right|\right\} .
\end{aligned}
$$

But we remark that, since $x=B_{K} \hat{x}+b_{K}$,

$$
\begin{aligned}
\int_{\partial K} x_{l}\left(\mathbf{v}-\bar{\Pi}_{K} \mathbf{v}\right) \cdot \mathbf{n} d \boldsymbol{\sigma}= & \int_{\partial \hat{K}}\left(B_{K} \hat{x}\right)_{l}\left(\overline{\mathbf{v}-\bar{\Pi}_{K} \mathbf{v}}\right) \cdot \hat{\mathbf{n}}\left|\operatorname{det} B_{K / \partial \hat{K}}\right| d \hat{\boldsymbol{\sigma}} \\
& +b_{K l} \int_{\partial \hat{K}}\left(\widehat{\mathbf{v}-\bar{\Pi}_{K} \mathbf{v}}\right) \cdot \hat{\mathbf{n}}\left|\operatorname{det} B_{K / \partial \hat{K}}\right| d \hat{\boldsymbol{\sigma}}
\end{aligned}
$$

Therefore,

$$
\begin{aligned}
\left|\int_{\partial K} x_{l}\left(\mathbf{v}-\bar{\Pi}_{K} \mathbf{v}\right) \cdot \mathbf{n} d \sigma\right| \leqslant & \left\|B_{K}\right\| \int_{\partial K}\left|\mathbf{v}-\bar{\Pi}_{\dot{K}} \mathbf{v}\right| d \sigma \\
& +\left|b_{K}\right|\left|\int_{\partial K}\left(\mathbf{v}-\bar{\Pi}_{K} \mathbf{v}\right) \cdot \mathbf{n} d \sigma\right|
\end{aligned}
$$


Since the last term is equal to 0 , we obtain

$$
\begin{aligned}
\left|\beta_{l}\right| \leqslant C & \left\{\left|\operatorname{det} B_{K}\right|^{-1 / 2}\left\|\mathbf{v}-\bar{\Pi}_{K} \mathbf{v}\right\|_{0, K}\right. \\
& \left.+\left|\operatorname{det} B_{K}\right|^{-1}\left\|B_{K}\right\| \sum_{i=1}^{4}\left|\operatorname{mes} F_{i}\right|^{1 / 2}\left\|\mathbf{v}-\bar{\Pi}_{K} \mathbf{v}\right\|_{0, F_{i}}\right\}
\end{aligned}
$$

so that, by Lemma II.3,

$$
\left|\beta_{l}\right| \leqslant\left\{h_{K}^{-3 / 2} h_{K}^{3}+h_{K}^{-3} h_{K} h_{K}^{7 / 2}\right\}|\mathbf{v}|_{3, K} \leqslant C h_{K}^{3 / 2}|\mathbf{v}|_{3, K} .
$$

The previous inequalities yield, for $k=0$ and 1 ,

$$
\left|\mathbf{v}-\Pi_{K} \mathbf{v}\right|_{k, K} \leqslant C h^{3-k}|\mathbf{v}|_{3, K} .
$$

By (II.8), the hypothesis (H3) is an immediate consequence of

LEMMA III.3. For any $\mathbf{v}$ in $H_{0}^{1}(\Omega)^{3}$, there exists $\mathbf{v}_{h}$ in $X_{h}$ such that

$$
\forall q_{h} \in M_{h}^{(2)}, \quad\left\{\begin{array}{l}
\left(q_{h}, \operatorname{div}\left(\mathbf{v}-\mathbf{v}_{h}\right)\right)=0 \\
\text { and }\left\|\mathbf{v}_{h}\right\|_{1, \Omega} \leqslant C\|\mathbf{v}\|_{1, \Omega} .
\end{array}\right.
$$

Proof. Let us denote by $\mathbf{w}_{h}$ the interpolate of $\mathbf{v}$ in the space

$$
\left\{u_{h} \in \mathscr{C}^{0}(\bar{\Omega}) \cap H_{0}^{1}(\Omega) ; \forall K \in \mathscr{T}_{h}, u_{h / K} \in P_{2}(K)\right\}^{3},
$$

defined by local regularization as in [1], so that (II.10) is still satisfied.

Then, we consider the element $\mathrm{v}_{h}$ in $V_{h}$ equal on $K$ to

$$
\mathbf{v}_{h}=\mathbf{w}_{h}+\sum_{i=1}^{4} \alpha_{i} \mathbf{p}_{i}+\beta \lambda_{1} \lambda_{2} \lambda_{3} \lambda_{4}
$$

with

$$
\begin{gathered}
\alpha_{i}=\left(\int_{F_{i}}\left(\mathbf{v}-\mathbf{w}_{h}\right) \cdot \mathbf{n}_{i} d \sigma\right) / \int_{F_{i}} \prod_{j=1, j \neq i}^{4} \lambda_{j} d \sigma, \\
\beta_{l}=-\int_{K} x_{l} \operatorname{div}\left(\mathbf{v}-\mathbf{w}_{h}-\sum_{i=1}^{4} \alpha_{i} \mathbf{p}_{i}\right) d x / \int_{K} \lambda_{1} \lambda_{2} \lambda_{3} \lambda_{4} d x .
\end{gathered}
$$

Clearly, one has $\forall q_{h} \in M_{h}^{(2)},\left(q_{h}, \operatorname{div}\left(\mathbf{v}-\mathbf{v}_{h}\right)\right)=0$. Moreover, by (II.6) and (III.7),

$$
\left\|\mathbf{v}_{h}\right\|_{1, K} \leqslant\left\|\mathbf{w}_{h}\right\|_{1, K}+C h_{K}^{1 / 2}\left(\sum_{i=1}^{4}\left|\alpha_{i}\right|+|\boldsymbol{\beta}|\right) .
$$

The $\alpha_{i}$ 's still satisfy (II.11). We also have

$$
\begin{aligned}
\left|\beta_{l}\right| \leqslant C\left|\operatorname{det} B_{K}\right|^{-1} & \left\{\left|\int_{K}\left(\mathbf{v}-\mathbf{w}_{h}-\sum_{i=1}^{4} \alpha_{i} \mathbf{p}_{i}\right)_{l} d x\right|\right. \\
& \left.+\left|\int_{\partial K} x_{l}\left(\mathbf{v}-\mathbf{w}_{h}-\sum_{i=1}^{4} \alpha_{i} \mathbf{p}_{i}\right) \cdot \mathbf{n} d \mathbf{\sigma}\right|\right\} .
\end{aligned}
$$


By the same way as in the proof of Lemma III.2,

$$
\begin{aligned}
& \left|\beta_{l}\right| \leqslant C\left[\left|\operatorname{det} B_{K}\right|^{-1 / 2}\left\|\mathbf{v}-\mathbf{w}_{h}-\sum_{i=1}^{4} \alpha_{i} \mathbf{p}_{i}\right\|_{0, K}+\left|\operatorname{det} B_{K}\right|^{-1}\left\|B_{K}\right\|\right. \\
& \left.\quad \times \sum_{i=1}^{4}\left|\operatorname{mes} F_{i}\right| h_{K}^{-3 / 2}\left\{\left|\mathbf{v}-\mathbf{w}_{h}-\sum_{i=1}^{4} \alpha_{i} \mathbf{p}_{i} \|_{0, K}+h_{K}\right| \mathbf{v}-\mathbf{w}_{h}-\left.\sum_{i=1}^{4} \alpha_{i} \mathbf{p}_{i}\right|_{1, K}\right\}\right] \\
& \leqslant C\left\{h_{K}^{-3 / 2}\left\|\mathbf{v}-\mathbf{w}_{h}\right\|_{0, K}+h_{K}^{-1 / 2}\left|\mathbf{v}-\mathbf{w}_{h}\right|_{1, K}+\sum_{i=1}^{4}\left|\alpha_{i}\right|\right\} .
\end{aligned}
$$

Finally, we obtain

$$
\left\|\mathbf{v}_{h}\right\|_{1, K} \leqslant\left\|\mathbf{w}_{h}\right\|_{1, K}+C h_{K}^{-1}\left\{\left\|\mathbf{v}-\mathbf{w}_{h}\right\|_{0, K}+h_{K}\left|\mathbf{v}-\mathbf{w}_{h}\right|_{1, K}\right\},
$$

which, together with (II.10), yields $\left\|\mathbf{v}_{h}\right\|_{1, \Omega} \leqslant C\|\mathbf{v}\|_{1, \Omega}$.

Consequently, this element can be used to solve the Stokes problem in the three-dimensional case with an $O\left(h^{2}\right)$-error estimate.

C.N.R.S. et Université P. et M. Curie

Analyse Numérique

4, place Jussieu

75230 Paris Cédex 05, France

C.N.R.S. et Université de Rennes

U.E.R. de Mathématiques et Informatique

Rennes Beaulieu

35042 Rennes Cédex, France

1. C. BerNARDI, “Optimal finite element interpolation on curved domains.” (To appear).

2. P. G. Ciarlet, The Finite Element Method for Elliptic Problems, North-Holland, Amsterdam, 1978.

3. P. G. Ciarlet \& P.-A. Raviart, "The combined effect of curved boundaries and numerical integration in isoparametric finite element methods," in The Mathematical Foundations of the Finite Element Method with Application to Partial Differential Equations (A. K. Aziz, ed.), Academic Press, New York, 1972.

4. P. Clement, “Approximation by finite element functions using local regularization," RAIRO Anal. Numér., v. 9, 1975, pp. 77-84.

5. M. Crouzeix \& P.- A. Raviart, "Conforming and nonconforming finite element methods for solving the stationary Stokes equations," RAIRO Anal. Numér., v. 7, 1973, pp. 33-76.

6. M. ForTIN, “Old and new elements for incompressible flows," Internat. J. Numer. Methods Fluids, v. 1,1981 , pp. $347-364$.

7. V. Girault \& P.- A. RaviaRT, Finite Element Approximation of the Navier-Stokes Equations, Lecture Notes in Math., Vol. 749, Springer-Verlag, Berlin and New York, 1979. 